\title{
1-YIA-23 YIA
}

\section{GPR143, a L-DOPA receptor, is involved in monocrotaline-induced pulmonary hypertension in rats}

\author{
Masayuki Nakano $^{1,3}$, Tatsuo Hashimoto ${ }^{1,3}$, Motokazu Koga $^{2}$, Daiki Masukawa ${ }^{1}$, Shinya Oku², \\ Yusuke Mizuno ${ }^{2}$, Takahisa Goto ${ }^{2}$, Kouichi Tamura ${ }^{3}$, Yoshio Goshima ${ }^{1}$
}

${ }^{I}$ Dept. Mol. Pharmacol. Neurobiol., Yokohama City Univ. Grad. Sch. Med., ${ }^{2}$ Dept. Anesthesiology Crit. Care Med., Yokohama City Univ., Sch. Med., ${ }^{3}$ Dept. Medical Science and Cardiorenal Medicine, Yokohama City Univ., Sch. Med.

We previously demonstrated that L-DOPA modulated the vascular $\alpha 1$-adrenergic receptor through GPR143, a Gprotein coupled receptor, and sensitized vasomotor tone. The purpose of this study is to clarify the involvement of GPR143, in pulmonary hypertension (PH). We generated GPR143 gene-deficient (KO) rats and comparatively studied monocrotaline (MCT) -induced PH in wild type (WT) and Gpr143-KO rats. We evaluated the interaction between L-DOPA and adrenergic $\alpha 1$ receptor by contractile force of rat isolated pulmonary arteries. The degree of $\mathrm{PH}$ was evaluated by right ventricular systolic pressure (RVSP) and right ventricular to body weight ratio (RV/BW). In isolated pulmonary arteries, L-DOPA $(1 \mu \mathrm{M})$ augmented contractile response to phenylephrine, an $\alpha 1$ adrenergic receptor agonist. One month after injection subcutaneously with MCT $(60 \mathrm{mg} / \mathrm{kg})$, the RVSP was attenuated in Gpr143-KO rats as compared to the WT rats $(49.7+/-1.1 \mathrm{mmHg}$ and $41.1+/-1.4 \mathrm{mmHg}$ in WT and Gpr143-KO, $\mathrm{p}<0.01, \mathrm{~N}=5$ ). Coordinately, the RV/BW was also reduced in Gpr143-KO rats compared to the WT rats $\left(5.8+/-0.3 \times 10^{-4}\right.$ and $4.9+/-0.2 \times 10^{-4}$ in WT and Gpr143-KO, $\left.<<0.05, \mathrm{~N}=7\right)$. We here provide evidence that GPR143 is involved in MCT-induced PH in rats. Further studies are needed to elucidate detailed mechanisms. 